\title{
Nuevas tareas, nuevos lugares del trabajo social judicial en España
}

\section{New tasks, new places of justice social work in Spain}

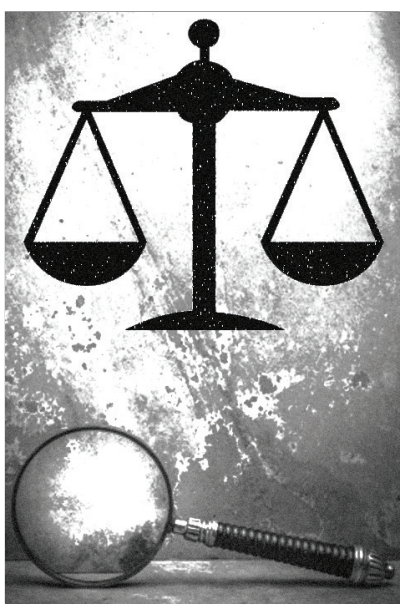

\section{Francisco Gómez Gómez* Raúl Soto Esteban ${ }^{* *}$}

\begin{abstract}
Resumen: Este artículo forma parte de una investigación cualitativa sobre el mapa de situación del trabajo social judicial. Una profesión con treinta años de vida en España y que se encuentra en revisión como todas las disciplinas y discursos en tiempos de crisis. Los relatos de los profesionales han dibujado una cartografía del trabajo social judicial dentro del equipo psicosocial que a su vez se sitúa dentro de los juzgados de familia.
\end{abstract}

Palabras-clave: Propuestas para Trabajo Social Judicial. Trabajo Social pericial.

\begin{abstract}
This article is part of a qualitative investigation about the situation of the justice social work. Such work has existed for thirty years in Spain, and it is being reviewed as every discipline and discourse in times of crisis. The professionals' reports sketched a map of the justice social work inside the psychosocial team that, in turn, is inside the family courts.
\end{abstract}

Keywords:. Proposals for Judicial Social Work. Expert Social Work.

* Doctor y licenciado en Ciencias Políticas y Sociología, diplomado en Trabajo Social, profesor titular de la Universidad Complutense de Madrid, España.E-mail: fgomez@trs.ucm.es.

** Trabajador social de los Juzgados de Primera Instancia e Instrucción de Alcobendas (Madrid), diplomado Universitario en Trabajo Social, licenciado en Historia del Arte. 


\section{Introducción}

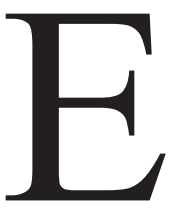

n los años en que esta investigación se desarrolla (desde el 2009 hasta la actualidad) la crisis económica y una serie de decisiones políticas posteriores han modificado en España la estructura del Estado de Bienestar. Las organizaciones y la disciplina del trabajo social han reaccionado ante la nueva realidad con protestas, el Consejo General de Colegios de Trabajadores Sociales redactaba una carta en la que protestaba por la situación derivada de la reforma de la administración local que retira la competencia de servicios sociales básicos a los entes locales: "La reforma, que también esconde privatizar los servicios sociales, pondrá en grave peligro la subsistencia de millones de familias, empujándolas a la exclusión y socavando sus derechos sociales y fundamentales." (Consejo General del Trabajo Social, 2013).

Pero también se reacciona con nuevas propuestas profesionales: el último Congreso de Trabajo Social de Madrid, organizado por el Colegio Oficial de Trabajadores Sociales de esta ciudad, se titulaba: "Trabajo Social Hoy: Nuevos contextos, nuevos compromisos, nuevos retos". En la ponencia marco, Cristina de Robertis describía la nueva situación:

Al mismo tiempo asistimos a una disminución de recursos financieros y humanos destinados a lo social en clara contradicción con el aumento de los problemas sociales y de las solicitaciones de las personas en dificultad. El imperativo es entonces hacer más con menos medios (Robertis, 2011, p. 11).

Plantea la misma autora los riesgos y las posibilidades de hacerles frente:

El trabajo social debe afrontar dos riesgos mayores: la mercantilización de la acción social y el retroceso social ( ) El trabajo social tiene que reanudar con prácticas inventivas, como lo fueron las de sus orígenes, apropiadas a los nuevos contextos y a los problemas actuales.

Hoy en día, el auge del trabajo social colectivo abre nuevas posibilidades de intervención a los trabajadores sociales. Una intervención más cercana de las personas, mejor articulada con la problemática actual y que facilite la inscripción 
de las personas en la sociedad y la adquisición de un rol ciudadano (Robertis, 2011, p. 20).

Aunque la autora se refiere a la realidad francesa, en la que trabaja, su reflexión sirve a los profesionales españoles. Más allá de esta reflexión cabría hacerse otra sobre la relación del trabajo social judicial con la intervención social y con estos nuevos roles que se plantean para el futuro. Reflexiones como la de pensar en como la realidad social en la que los trabajadores sociales suelen intervenir es la de los individuos con carencias; no es el caso de los trabajadores sociales en los juzgados de familia, los cuales evalúan situaciones de crisis familiares. Las relaciones familiares se han deteriorado hasta el punto de llegar a la ruptura, situación propia de todas las clases sociales. Desde los profesionales del trabajo social judicial en Buenos Aires lo han descubierto y trabajado:

Una primera distinción ha sido reconocer el desafío de intervenir sobre problemáticas que no eran fruto de condiciones socioeconómicas deficitarias (en las que nuestra formación académica ha centralizado el foco) sino fruto del complejo entramado vincular que producía enfrentamientos entre los miembros de una familia que, en buena parte de los casos, parecía inacabable o inclusive aumentada por la presentación ante un tribunal (Alday, Ramjlak y Nicolini, 2001, p. 91).

Todo ello dentro de una definición genérica de la competencia profesional que se realiza en la creación del libro blanco y que edita la ANECA (Agencia Nacional de Evaluación de la Calidad y Acreditación):

El/la trabajador/a social es un/a profesional de la acción social que tiene una comprensión amplia de las estructuras y procesos sociales, el cambio social y del comportamiento humano, que le capacita para:

- Intervenir en las situaciones (problemas) sociales (de malestar) que viven individuos, familias, grupos, organizaciones y comunidades, asistiendo, manejando conflictos y ejerciendo mediación.

- Participar en la formulación de las Políticas Sociales.

- Contribuir a la ciudadanía activa mediante el empoderamiento y la garantía de los derechos sociales (ANECA, 2005, p. 111). 


\section{Metodología}

Este artículo se sirve de los resultados de técnicas cualitativas de análisis de la realidad social, en este caso de una realidad profesional. Las técnicas son las entrevistas en profundidad y los grupos de discusión. El resultado es un conjunto de discursos de jueces y trabajadores sociales, en el primer caso a través de entrevistas individuales a jueces de familia de Barcelona y Madrid. Y respecto al segundo grupo de profesionales: se realizan entrevistas a trabajadores sociales judiciales de Barcelona y Madrid y se realizan dos grupos de discusión en la Comunidad de Madrid.

La intención es exploratoria, dibujar un mapa, hacer un recorrido por la profesión. Y el último destino de la travesía es la visión que los profesionales tienen sobre el futuro, la de los propios trabajadores sociales judiciales y la visión de los jueces de familia, destinatarios de su trabajo. La mirada superficial, aérea se nutre de discursos de los profesionales de Madrid y Barcelona que configuran una idea de la situación de la profesión en España.

\section{Resultados}

La intención de la exploración, del estudio es ver nuevas utilidades y funciones, lo cual es el contenido obvio de las respuestas. Pero también lo es la visión futura del trabajo la que interesa, y si los jueces, en primer lugar, ven futuro en la tarea profesional de este colectivo, y en que sentido dirigen ese futuro.

En las distintas entrevistas con jueces se aprecia una diferencia entre las entrevistas con profesionales de Madrid y las efectuadas a jueces de Barcelona. En estos últimos late de forma clara una circunstancia contextual que es necesario reseñar: se les ha notificado por el órgano correspondiente de la Generalitat de Cataluña que sus equipos psicosociales (SATAF) van a elaborar sólo informes para partes de los procedimientos que cuenten con el beneficio de la justicia gratuita, situación resultado de una saturación del servicio. Estos jueces insisten en la escasez de medios de forma más clara que los 
jueces de Madrid. En los jueces de Barcelona se hace mención a la necesidad de contar con una mayor cercanía y compromiso de los equipos psicosociales, mientras en los de Madrid, que cuentan con el servicio más cercano, no se da esta queja.

Se recogen reivindicaciones en dos jueces que piden la figura del psiquiatra como necesario en los equipos psicosociales. En este sentido se cita primero la aportación de los jueces de Madrid, en los cuales se expresa una mayor cercanía y un cumplimiento más cercano a los deseos del juez. Se les pregunta de forma genérica por nuevas tareas o roles a ejercer por los trabajadores sociales judiciales:

Vaya pregunta (risas), nos centramos en familia, así que se me ocurre sobre la marcha, cuando habláis con las familias ya les asesoráis sobre los servicios que pueden adecuarse a sus circunstancias, que seáis un canal, es lo que porque hay un desconocimiento Aparte del asesoramiento al juzgado, yo creo que lo hacéis. Ahora no se me ocurre que más (cita de J1M). ${ }^{1}$

La mediación es una función que los jueces ven importante y algunos la proponen como función de los equipos psicosociales. También se deja ver que es bien recibida la aportación sociofamiliar y se reseña la demanda de más recursos:

Soy consciente de que el tema de mediación no se puede hacer, yo echo de menos mediación dentro del juzgado. Tendría que haber más gente, más equipos, tenéis mucha experiencia y que el que mediase no viese a la familia. Tenéis mucha experiencia, sería muy importante. Por lo demás, lo de las incapacidades que te he comentado. Para mí ha sido bueno que hicieseis cada uno el informe, que al principio me chocó muchísimo, pero ha sido enriquecedor que hicieseis cada uno el suyo, así he visto que es de trabajador social y que del psicólogo. Y no sé. Necesitamos más equipos, con más formación, echamos en falta tema organización, temas materiales que os faltan (cita de J2M).

1. Las citas de las entrevistas se refieren de forma anónima a la profesión ( $\mathrm{J}=\mathrm{Juez}$ y TS $=$ Trabajador Social), al número de orden en ser entrevistado y al lugar de procedencia del entrevistado: Madrid o Barcelona). 
En algunos casos (en Madrid, capital) hay una colaboración directa: cada juzgado tiene su propio equipo y la coordinación está más integrada.

Yo es que parto de lo que dije al principio, yo como estoy muy satisfecha con el trabajo de mi equipo, es que no se me ocurre qué más pueden hacer, porque lo hacen todo. Todo lo que a mí se me ocurre, o a ellas se les ocurre y me proponen, se hace. Yo creo que lo que faltaría es aumentar los equipos, y que cada juzgado tuviera su equipo, y que cada equipo contara con un psiquiatra sería mi principal petición (cita de J4M).

En los jueces de Barcelona se produce una necesidad de mayor compromiso y cercanía, se llega a decir que el trabajador social no debe ser un profesional para las partes en el conflicto y que el rol debe ser educativo. Hay comparaciones con sistemas europeos y nuevas experiencias. También se habla de un nuevo derecho con nuevas búsquedas:

Hombre, yo creo que en esta parcela de la jurisdicción hay que trabajar mucho en medidas educativas, en medidas de entendimiento y comprensión de los hechos a todos los niveles. Estamos viviendo el paso de la justicia de la persona o la familia, que se usaba el sistema patrimonial a una jurisdicción diferente en la que los jueces en vez de juzgar el pasado se establezcan normas para el futuro. Y entonces si los jueces han de hacer normas para el futuro de las familias, auxiliarse de los especialistas en esta materia es fundamental y se ha de hacer, y hemos hablado de cómo los equipos hacen informes y están muy separados del día a día de la familia, o de momento que los equipos intervendrán más tranquilamente, por supuesto no intervendrán siempre, es importante, hay juzgados que en cuanto la parte pide el informe lo acuerdan, aquí nunca se ha hecho. Si la parte puede sugerir que intervenga, pero es el tribunal el que decide, y si deriva solo en los casos que el juez necesita, pues sí, hay posibilidad de hacer más cosas. En ocasiones la presencia del trabajador social, y del psicólogo, en la exploración de menores, o la presencia en interrogatorios de las partes, cuando el trabajador social ya ha tenido las entrevistas previas y ya conoce la personalidad, puede enriquecer mucho la tarea (cita de J1B).

Se propone que los equipos psicosociales no sean peritos de las partes, sino profesionales que ayuden al juez y a través de él a las familias. Es funda- 
mental en esta propuesta un trabajo cercano e integrado entre jueces y técnicos. Se compara con Francia la situación actual:

Mientras se de un perito al servicio de las partes, es desde mi punto de vista un error, se implantó el sistema de los equipos psicosociales de manera provisional, de manera piloto para tener una valoración, y en ese sentido en Francia, pero nunca se ha hecho por el legislador esa reflexión y como va sobre la marcha en los tribunales va a depender mucho de las experiencias de los jueces, de si se lleva bien con su psicólogo, con su trabajador social, de si han confiado en ellos, porque algunos que les hablen de usted, les hacen cuadrarse, les amenazan con meterles en cárcel. Hay otros que asumen que es un colaborador estrecho y creo que ese es el camino (cita de J1B).

Los jueces refieren aspectos de su experiencia profesional y recorren y describen la relación con otras realidades europeas con las que han tenido contacto, destacando el trabajo extrajudicial como fundamental para reducir la "litigiosidad" y los conflictos. También se produce un mayor respeto a la autonomía de la familia a la hora de resolver sus propios conflictos:

Te voy a decir una cosa: la importancia de los trabajadores sociales en otros países es fundamental en el ámbito de familia. Por lo que tengo entendido, en Alemania, por ejemplo, cuando los padres se divorcian si hay acuerdo en relación con las medidas personales de los hijos, los padres no van al juzgado para nada, fijate que poco intervencionista es aquél sistema. Los padres son los padres, es su tema, ni fiscal ni nadie más (cita de J2B).

Cuando la situación les supera existen órganos administrativos que tienen técnicos para solventar la situación con intervención directa, y si no logran el acuerdo estos profesionales actúan en las salas de juicio, sobre todo respecto a los sistemas de visitas.

Si el problema es en relación con el régimen de visitas no van directamente al juez, sino que en los "Jugendamt" (oficina de la juventud), hay trabajadores sociales y psicólogos encargados de los regimenes de visitas de los padres separados. Estos trabajadores sociales intervienen activamente en el conflicto, se implican, 
siempre desde la distancia profesional, pero actúan de forma activa, dando alternativas, viendo de mejorar la relación, encontrando soluciones concretas al caso. Tratan a las partes como un profesional amigo. Sólo para el caso de que el problema no se pueda resolver por esta vía las partes van al Juzgado a plantear sus controversias. Entonces, los trabajadores sociales siempre intervienen en los juicios en los cuales el objeto es el régimen de visitas. Siempre están en el acto de la vista, necesariamente, para orientar al Juez y a las partes también. Ayudan mucho. Oyendo a las partes implicadas y al trabajador social que ha intervenido en el caso siempre es posible alcanzar acuerdos (cita de J2B).

También destacan la diferencia de recursos, cuando hay un menor volumen de trabajo los juzgados son más ágiles, pero la mentalidad colectiva que busca el acuerdo también reduce el tiempo de los procedimientos. Y destaca el rol del trabajador social:

En Alemania, es raro el juicio que no termina en un acuerdo, en el ámbito del derecho de familia. Claro que es importante el hecho de que tienen muchos menos asuntos (unos 400 asuntos por Juez al año, en los Juzgados de Familia de Berlín, aquí en Barcelona, tenemos unos 1.200 asuntos al año por Juzgado). Por este motivo no tienen un límite de tiempo tan estricto en cuanto a la duración de las vistas. Pueden dialogar y dialogar, hasta alcanzar el acuerdo. Pero, en cuanto a lo que tú preguntabas, que era sobre el papel del trabajador social, pues fíjate que en el sistema alemán es fundamental, fundamental, pues se trata de una persona que actúa como un observador objetivo en el conflicto, que puede reconducir la situación, o bien antes del juicio o bien en el mismo acto del juicio. La implicación del profesional también es esencial, como siempre sucede en cualquier función que se realice (cita de J2B).

Los jueces piden una mayor neutralidad en los procedimientos en que haya una oposición a una decisión administrativa, que no se den por buenos directamente informes de profesionales de la administración por tener esta condición.

Insisto en lo del psiquiatra. Insisto en lo del psiquiatra, también en una mayor neutralidad frente a los procedimientos de oposición de la administración. Es una cuestión de tener cuidado que es una administración y que es otra administración. 
Y para demostrar que esto no es así, ustedes tendrían que tratar con el mismo cuidado que cuando hay dos particulares, y contrastar criterios, y no dar por buenos las afirmaciones de la administración en ningún momento (cita de J3B).

Hay una necesidad ya creada en los procedimientos de familia por esta nueva visión de la justicia que ya se ha apuntado. Donde antes existía una visión del juez que tenía poco margen de interpretación o decidía directamente, ahora se produce una mayor discrecionalidad en sus decisiones y una influencia de los jueces en las vidas familiares que les hace necesitar la ayuda técnica en mayor medida.

El problema es de medios, no es un problema de no hacer las cosas correctamente. El SATAF es percibido por los abogados como un órgano que tiene demasiada importancia, y está que la propia naturaleza de la pericial de un psicólogo, y como he dicho al principio, imparcial y objetiva, es muy importante, y a pesar de esta opinión que puedan tener los abogados, la verdad es que tal y como se hacen los informes del SATAF son suficientes, no creo que se pueda hacer o acusarles de partidismos. El problema es lo que tarda el SATAF. Ahora el SATAF no está haciendo informes porque no tiene medios y está colapsado, los informes no se hacen. El problema es de medios, y el SATAF es muy importante, pero si no es el SATAF tendrá que ser otro servicio u otro profesional, porque es muy importante (cita de J4B).

La misma pregunta se realiza a los trabajadores sociales de Madrid y Barcelona. Se aprecia una diferencia que responde a un distinto momento profesional, los profesionales de Madrid se centran en la necesidad de investigar en la tarea realizada (salvo algún trabajador social que incidiría en el mismo tema que sus compañeros barceloneses): en valorar los casos de custodia compartida, en evaluar que sucede con las adopciones por matrimonios y parejas homosexuales, en avanzar en la prevención o en la coordinación entre profesionales. Mientras, los trabajadores sociales judiciales de Barcelona buscan el lugar dentro del juzgado y de la administración, y su forma de responder a la pregunta sobre nuevas tareas es profundizar en ser trabajadores sociales, en usar nuestro léxico y nuestras técnicas y en no evitar el trabajo social o sociofamiliar, 
en un expediente sin participación del psicólogo, a pesar de que se prefiera el trabajo en equipo.

Se recoge la opinión de los profesionales de Madrid en primer lugar, los cuales quieren investigar, conocer que pasa con las nuevas realidades (custodia compartida y adopción en parejas homosexuales sobre todo) pero no a corto plazo, sino en estudios que dibujen el futuro de las nuevas dinámicas familiares.

Si yo creo, no sé, se pueden hacer muchas cosas por ejemplo de investigación y, hacer algún tipo de estudios sobre una medida que se ha tomado cosas que se van modificando, que va entrando la sociedad, por ejemplo las guardas y custodias compartidas. Sería muy interesante que por ejemplo con el paso de dos años hacer un estudio de cómo se desarrolla, porque estamos haciendo cosas sin saber las consecuencias. Eso es lo que me produce realmente malestar, pero si lo supiésemos como lo van a saber dentro de unos años, como valorar. Te voy a poner un ejemplo: el tema de adopciones en parejas del mismo sexo, yo no valoro si es malo o es bueno, yo hago mi trabajo como están esas relaciones y punto. Pero a mí sí que me gustaría saber cómo interfiere eso, cómo va a ser, como se va a desarrollar un menor y si es distinto a cómo es la mayoría, no porque sea bueno ni malo, sino simplemente que matices hay y como se van a desarrollar, cómo se sienten los menores, y seguramente se sentirán bien, esa es mi teoría, pero no sé, pero no lo sé, por la sociedad, sabes, no sé cómo van a ser recibidos o cómo ellos se van a sentir como individuos (cita de TS1M).

Se aprecia una falta de estudio de una realidad en transición, de forma que se produce poco discurso ya que no se investiga y no se tienen datos, tan sólo hipótesis sin contrastar. Además de la falta de un discurso teórico que fundamente la acción, falta una implicación con los profesionales de los servicios con los que las familias se relacionan.

Yo creo que nos ha faltado implicarnos en haber hecho un análisis de todo este trabajo que venimos haciendo y haber buscado desde lo social una complementación. Pero yo, vino el PEF (Punto de Encuentro Familiar) que genial por las complicaciones, pero creo que debería trabajarse desde la prevención. No sé si no hemos hecho campaña a nivel de barrio, cuando se hicieron los CAI (Centros 
de Atención a la Infancia). En la red de servicios sociales haber hecho prevención. El haber dado otra categoría a la mediación familiar desde la red social. Yo no sé si todo el mundo ha tenido acceso a los centros de mediación. Trabajar mucho a todos los niveles: mediación, prevención hasta en las escuelas (cita de TS2M).

La falta de recursos y el carácter puntual de la intervención hacen imposible estudios transversales basados en una intervención propia, sólo cuando se acuerdan seguimientos cabe esta posibilidad.

A nivel de familia, sería muy conveniente el hacer un seguimiento de algunos casos, pero a nivel profesional, que se hace pero llevarlo a cabo como me gustaría no puede ser. Aunque esté estipulado que lo hagamos, pero no porque lo diga una sentencia, sino por interés y tarea profesional.

Luego el tema de coordinación no tenemos bastante (cita de TS6M).

Hay también en los profesionales de Madrid una preocupación, menos general pero real, en el lugar de la profesión y por ello plantean en el futuro estudiar ese lugar y esa organización actual:

Por ejemplo (...) no tenemos la necesidad de que nos pregunten sobre el informe psicosocial. Va más para el psicólogo. No sé porque es, si porque pensamos que decimos cosas menos interesantes, si a los abogados les da más juego lo psicológico porque empiezan con los test, no sé, pero bueno es algo que deberíamos pensar. Por la importancia que deben tener los trabajadores sociales. Y, después, de cara a las familias, es curioso, porque yo en algún caso, por alguna circunstancia he visto sola a una familia, y no tengo ningún problema, voy incluso a la ratificación y no pasa nada, he trabajado individualmente y no he tenido ningún problema. Se ha hecho en el equipo el reparto del caso y luego hemos visto sin problema, (...) pero da la impresión que en el trabajo en equipo, cobra más importancia el trabajo del psicólogo (cita de TS3M).

Los trabajadores sociales refieren su preparación para realizar el trabajo en solitario, para evaluar una familia, y siguen viendo que al trabajar en equipo su rol pierde fuerza: 
No sé si es algo, aunque en el equipo vamos a la par, parece que al ser un rol superior en cuanto a conocimientos técnicos, es un poder el que se le otorga. Aunque luego es verdad, que en el contacto con la familia, con los jueces yo no he sentido eso. Pero sí que se percibe así. Muchas veces yo tengo que decir que soy trabajadora social, y siguen diciendo la psicóloga a mí me atribuyen eso. Pero por qué, y te presentas y lo dices, pero continuamente están y no sé por qué. Eso yo lo veo así y lo he hablado con compañeros. Si trabajamos individualmente les obligamos a evitar eso, pero yo creo que si podemos trabajar en equipo y que no lo debemos perder. No sé como se puede arreglar. Es difícil pero tengo las dos experiencias y defiendo trabajar en conjunto. Pienso que le damos juego entre comillas, da más marcha en la disputa judicial la psicología. En algunos casos, como los de protección, yo ahí veo que recuperamos el centro y lo ves en la sala, con la familia, en la familia disfuncional. Sin embargo en otras familias (cita de TS3M).

Mientras en los de Barcelona se observaría esa tendencia a buscar el lugar del trabajador social, su posición en el equipo y su reforzamiento como figura profesional, esta sería la tarea futura más acuciante para ellos. Dentro de esta búsqueda se destaca un valor propio de la profesión: el de potenciar a las familias, el de buscar recursos y potencialidades en lugar de grietas y defectos, carencias o patologías. Dicho de una forma constructiva: "Cuando la intervención del trabajador social está orientada a evaluar los vínculos dentro de una organización familiar y acompañar a la familia a reorganizar su dinámica atendiendo las necesidades de todos sus miembros, es más posible operar para el cambio" (Robles, 2004, p. 99). Esta puede ser una buena diferencia y a la vez una aportación profesional.

Ya mucho de lo que hacemos parte de nuestra profesión, de ayudar a tener beneficios en el otro. No "negativizar" capacidad sino buscar potencialidad en la familia, en los miembros. Al menos aquí en Barcelona que es donde yo tengo la experiencia, a nivel de trabajo social se ve bastante, como en los informes, de intentar; si que tenemos que decidir, eso no, es el juez, pero sí valorar una situación y decidir lo mejor para el niño, pero sin entrar en la batalla en la que están los progenitores. Sin entrar a ajusticiar a uno de ellos, sino a buscar lo que hacen bien cada uno de ellos, yo creo que es la principal tarea desde el ámbito de la 
justicia, donde el contexto es bastante tóxico de pelea, de lucha de binomio ganadores/perdedores, y un poco la orientación es todos ganamos o todos perdemos, pero el que peor lo va a pasar es el niño. O nos ponemos todos las pilas o por mucho que gane uno a nivel judicial estamos perdiendo todos (cita de TS1B).

La tarea puede ser la de buscar un proyecto y como se decía antes preparar el futuro de las familias. Y dentro de este trabajo aportar la versión más propia sin complejos.

Muchas veces nosotros decimos lo que hay detrás de las familias, independientemente de la medida judicial que se adopte, el problema persiste. O sea, porque es un engranaje familiar y una medida judicial puede que no afecte, aquí si que aportamos esta visión, mas de potenciar esto. Por mucho que sea o custodia compartida o exclusiva con un amplio régimen de visitas, tenéis que buscar esto, estar pendientes de los hijos, esto es un puntal y ya se hace y que tendríamos que perseguir más. Y utilizar más lenguaje nuestro propio, a mi me hace gracia, nosotros hablamos del desarrollo psicoemocional del niño, y yo ya llevo un tiempo con el desarrollo socioemocional del niño, yo le pongo socio siempre que puedo ( ). Y el informe sociofamiliar. Yo creo que en esto podemos hacer un poco más. Poner un poco o la diferenciación porque te puedes encontrar que en los juicios, nos ha pasado, te puedan recusar o intentar recusar por ser trabajadora social (cita de TS1B).

La situación de los equipos psicosociales en Cataluña ha pasado por varias etapas debido a la dependencia de los profesionales de una administración pública o una ONG, esto ha afectado a la profesión de los trabajadores sociales y a que en la actualidad las profesionales sean menos en número que los psicólogos. Pero la actualidad se describe como un momento de fuerza y de iniciar una nueva etapa:

Mi opinión es que eso pasó en el momento en que Generalitat volvió a asumir las competencias y la gestión del servicio. Yo creo que no es por una cosa, es un cúmulo de situaciones y de motivos que llevaron a eso, fue un momento en que había muchas críticas, pero no muchas más de las que podrían haber ahora, no sé, por la cohesión del equipo, y más cuestionamientos a la figura del trabajador 
social, con lo cual te veías más en la necesidad de justificarte, el porqué tú si eras válido para hacer eso, la valoración de esa familia, también creo que en aquel momento, y eso es una cosa como muy personal, el conjunto de trabajadores sociales de momento no era lo suficientemente fuerte para plantear eso, con lo cual delante de la debilidad, se decía mira, pues si éste me está diciendo esto, pues yo no lo hago porque me van a cuestionar, mira he ido a un juicio y me han machacado porque era trabajador social, y al final no sabía como defenderme, y entonces te planteas, a lo mejor necesito una psicóloga. ( ) Y ahí está y pesa, pero lo que pasa es que el trabajo social ahora es más fuerte que en aquel momento. Es un colectivo muy fuerte, habrá quien se lo crea más y quién se lo crea menos y es ahora más fuerte, y las críticas nos hacen menos daño (cita de TS2B).

La necesidad de una mayor publicidad del trabajo y la nueva titulación de grado se perfilan como ayudas para la nueva etapa.

Muchas cosas yo creo, pero el trabajador social yo creo que nos hemos creído que estamos en inferioridad, no y eso hace, bueno es que tenemos una diplomatura, ahora cambia. La idea es está y como que te va calando, calando y no sacamos provecho de nuestra profesión. Yo, por ejemplo, tenemos ahora un espacio desde el colegio en un programa de radio, y los trabajadores sociales podemos hablar durante quince minutos, y yo ahora voy a hablar de nuestra profesión, he empezado a mirar y nuestra profesión es una pasada, pero claro he necesitado mirármelo para recordar. ( ) La propia profesión tiene términos que son negativos, no hablamos de inclusión, y sí de exclusión, marginalidad, contextos. Son palabras que ya son lo peor, los trabajadores sociales llevan lo peor, entonces claro, no tiene porque ser así. Y también porque se ha trabajado mucho a nivel individual, pienso que a nivel colectivo se pueden obtener mejores resultados. Entonces yo creo que nosotros no nos sabemos vender, ese es el problema (cita de TS3B).

También se plantea a los grupos de discusión de trabajadores sociales la cuestión de las nuevas tareas de los trabajadores sociales. Los trabajadores sociales sí encuentran nuevas tareas a realizar, y su inquietud va dirigida a ampliar la función pericial, ir más allá de la función evaluadora y seguir la marcha de la familia, en su trayectoria en contacto con los recursos del entorno. 
3. Sí esto, porque luego te vuelve. Haces un informe y al cabo de un año vuelve.

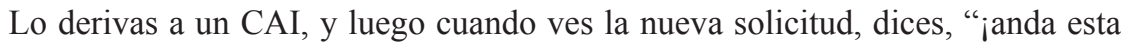
familia otra vez!”. Además es que te quedas como vacío, has estado trabajando con una familia, viendo y después nada, esperas lo mejor, por supuesto, pero pasado el tiempo, vacío.

1. Es como lo del menor, que hacen la mesa del menor. Esa necesidad yo la he tenido en determinados casos, que son los más complicados, que intervienen muchos profesionales, que el juez atiende tu propuesta y luego te planteas: ¿qué habrá pasado de esta familia o esta mujer? Y a mí me queda la propuesta de hacer una mesa sobre esto, para ver si esta propuesta que he hecho ha servido para algo o qué. Ha sido un descalabro y no ha servido para nada. Sería una mesa de familia.

2. Soltar el caso es lo más complicado. Cuando vienes de otros ámbitos te cuesta, porque te vienen, los conoces. Le das el alta (cita GDTS2). ${ }^{2}$

Los trabajadores sociales discuten también sobre la función de control de las familias que puede atribuirse a los trabajadores sociales en un rol discutible. La nueva justicia que como se indicaba anteriormente demanda a los jueces la tarea de organizar las familias, puede querer controlar las consecuencias de esas decisiones lo que daría garantías técnicas, pero situaría a las familias en un lugar público permanente y su autonomía se cuestionaría. El debate se abre. Robles (2004) describe con acierto el cambio en las familias:

Cuando la "familia completa" a la que aludíamos se ve afectada por la crisis de la separación o el divorcio, se desmoronan los pilares ideológicos que la sostenían y un estado de desconcierto e incertidumbre parece apoderarse de sus integrantes. Aquella familia, antes comprendida como "normal" puede ser catalogada como "problema", pudiéndose verse seriamente afectados los procesos que en ella se desarrollan. Es allí cuando (re)aparece en escena y protagónicamente el Estado, representado a través de las organizaciones que integran uno de sus poderes, los juzgados. Es en ese tránsito de las familias por la Justicia donde lo público y lo privado se entrelazan; ecuación que devendrá articulada o excluyente, complementaria

2. Las citas de los grupos de discusión agrupan una o varias opiniones o discursos, en primer lugar se cita el grupo, los profesionales que lo forman: trabajadores sociales y el número de grupo cuando hay varios. Cada número distingue el discurso de un participante diferente dentro del grupo. 
o suplementaria, aunque siempre conflictiva, siendo su resultado la síntesis de un proceso complejo, a veces atravesado por el estereotipo y, otras, por la creatividad (Robles, 2004, p. 31).

En el camino en el que la actuación profesional puede introducirse en al ámbito privado y condicionar la capacidad de la familia y su propia autonomía, los profesionales deben recordar su rol de ayuda a las familias, antes que su propia autoestima profesional o su deseo de colaborar con el poder de la Justicia a costa del bienestar familiar.

3. Yo quería comentar que hice un curso en la UNAF (Uninión de Asociaciones Familiares) y vino una americana con sus novedades yanquis. Parece ser que está proliferando una figura en EEUU que es el controlador parental, muy vinculado a los juzgados porque es el juez el que dice u obliga a las partes a ir al controlador parental y ese controlador parental hace informes para los jueces. La verdad es que era curioso. En "plan coña", decíamos como "policía parental". En caso de incumplimiento de régimen de visitas, en situaciones extremas, que me parece fenomenal. Ella decía que hacía mediaciones, pero era una "chivata judicial", pero en familias concretas es la única solución, que te van a dar. Mira lo que puede pasar. Si dentro de los equipos hubiera tiempo, podría estar bien. Si remites al controlador parental estaría bien.

1. Yo en Barcelona, hacía de chivato, trabajador social, más terapeuta, más todo. "Que lo reinicie". No existía recurso para reiniciar, pero se hacía. ( )

2. Aquí se hace todavía.

1. Y se cronifica mucho el problema. Todo tiene que tener un final y que el juez tome una decisión. Si no toma la decisión, se cronifica.

2. Y es muy cómodo para los padres.

1. No, para los jueces.

2. Sí, pero pasa también con los padres con el punto de encuentro, se cronifica y se tiran allí. Para uno de los padres, incómodo, para el hijo, incómodo. Pero para los padres es cumplir con lo escrito y ya está. En familia en Madrid, desde los juzgados de familia se cubría esa necesidad por los profesionales. Eran las trabajadoras sociales las que iniciaban el régimen de visitas en el fin de semana. Se iban al retiro o lo que sea para empezar el régimen de visitas (debate de GDTS1). 


\section{Conclusiones}

— El mapa de futuro de la actividad profesional de los trabajadores sociales judiciales tiene una situación diferente en los dos territorios estudiados: en Barcelona existe una clara preocupación por el futuro del SATAF y de la asistencia que presta. En Madrid no hay tal incertidumbre y la situación es más estable, a la vez que la relación entre los equipos psicosociales y los jueces es más cercana.

- Los jueces de Barcelona piden más recursos y una visión distinta de los equipos psicosociales, ellos creen que se debe adoptar una actitud de cercanía y ayuda a las familias, una mayor dedicación a las labores de prevención y mediación para evitar el exceso de intervención judicial. Todo lo integran en una justicia distinta en la que se pide a los jueces que organicen el futuro de las familias, y por tanto los jueces deben contar con ayuda técnica y a la vez respetar la independencia de las familias y buscar que sean autónomas en esta organización.

— Los jueces de Madrid entrevistados se muestran más optimistas con la relación que tienen con sus equipos psicosociales y sus trabajadores sociales. Se aprecia una mayor integración del trabajo y las demandas para el futuro son de más recursos, introducir la figura del psiquiatra y la mediación familiar entre las funciones de los trabajadores sociales de cada juzgado.

- Los trabajadores sociales de Barcelona se centran en su propio lugar en el mapa profesional de la Administración de Justicia. Tras una serie de avatares profesionales, por los que han pasado, analizan su situación actual, y desde una perspectiva asertiva como grupo, creen que hay que centrarse en lo propio y desarrollar un discurso de trabajadores sociales y plantear una actitud profesional más centrada en potenciar a las familias que en marcar sus condiciones deficitarias, dentro de una lógica de "empoderamiento", integrada en la competencia y el perfil profesional.

- Los trabajadores sociales de Madrid desarrollan un discurso más centrado en la tarea y plantean como necesaria la investigación de los nuevos tipos de familia (inmigración, hijos de padres homosexuales, custodia compartida ). A la vez se expresa la dificultad de este trabajo por el carácter puntual del 
trabajo y la falta de apoyo institucional. También se plantea en los grupos de discusión el rol de controlador del trabajador social en los seguimientos del régimen de visitas, como una función histórica y discutible.

Recebido em 16/6/2014 - Aprovado em 24/11/2014

\section{Referencias bibliograficas}

ALDAY, M. A.; RAMJLAK, N. L.; NICOLINI, G. M. El trabajo social en el servicio de justicia. Buenos Aires: Espacio, 2001.

CONSEJO GENERALDE COLEGIOS OFICIALES DE TRABAJADORES SOCIALES. Disponible en: <http://www.cgtrabajosocial.com/noticias/>. Acceso en: 26 jul. 2013.

ROBLES, C. La intervención pericial en Trabajo Social. Buenos Aires: Espacio, 2004. VV. AA. Libro blanco del título de grado en trabajo social. Madrid: ANECA, 2005. 